\title{
La perspectiva de la sociedad en las evaluaciones económicas en salud en el Perú
}

\section{The societal perspective in the health economics evaluation in Peru}

Correspondencia

Seimer Escobedo Palza

sescobedope@yahoo.com

Recibido: 27/09/2016

Aprobado: 28/og/2016

Citar como: Escobedo-

Palza S, Timaná-Ruiz R. La

perspectiva de la sociedad en las evaluaciones económicas en salud en el Perú. Acta Med Peru. 2016;33(3):261-2

\author{
Seimer Escobedo-Palza'1 , Raúl Timaná-Ruiz² \\ 1 Sociedad Peruana de Administración en Salud. Lima, Perú. \\ 2 Instituto de Evaluación de Tecnologías en Salud e Investigación, EsSalud. Lima, Perú.
}

\section{Sr. Editor:}

Actualmente las evaluaciones económicas en salud (EES) se utilizan cada vez más en la toma de decisiones ${ }^{[1]}$ aplicándose en la formulación y evaluación de políticas de salud, en la gestión y financiamiento de la atención sanitaria así como en las decisiones clínicas.

Las EES se definen como "el análisis comparativo de los cursos alternativos de acción tanto en términos de sus costos, como de sus consecuencias" [i] y se pueden clasificar en parciales o completas. Las primeras evalúan solo los costos o solo las consecuencias; mientras que las segundas evalúan tanto los costos como las consecuencias ${ }^{[2]}$. Las EES se pueden realizar desde diferentes perspectivas, siendo las más utilizadas: del financiador, del prestador, del paciente y de la sociedad ${ }^{[1,2]}$.

La perspectiva de la sociedad (PS) incluye la identificación y valoración de todos los costos de los recursos asociados con el uso de una intervención sanitaria ${ }^{[3,4]}$. Las demás perspectivas son subconjuntos de la PS; por tanto, pueden ser utilizadas en una misma EES [4]. La PS incluye costos directos sanitarios, los no sanitarios y los costos indirectos, también llamados costos de productividad. La importancia de la PS radica en la valoración de los costos no remunerados, como los costos por cuidado familiar o los costos de un personal para el cuidado de una persona enferma, esto reviste de mucha importancia en enfermedades crónicas y discapacitantes ${ }^{[5]}$. Asimismo, la valoración de los costos de productividad son una marca registrada de la perspectiva social, estos se refieren a los costos que una persona deja producir por una enfermedad, especificamente hace referencia al coste de oportunidad que una persona deja de percibir por estar enferma o por muerte. Para la estimación de los costos de productividad se utilizan diversas metodologías, las más usadas son el enfoque del capital humano y el método de los costes de fricción ${ }^{[6]}$.

En distintos manuales y guías metodológicas la PS es la más recomendada, ya que en teoría facilitaría el diseño de políticas dirigidas a la maximización del bienestar de la sociedad. Sin embargo, se reconocen dificultades prácticas para realizar estudios con la perspectiva de la sociedad, y por lo general se emplean puntos de vista más restringidos $\left[{ }^{[7]}\right.$.

Un estudio del año 2009 evaluó los costos y la perspectiva de los estudios de costo efectividad publicados; sus hallazgos fueron que un $40,6 \%$ de los estudios habian sido realizados con la PS, en contraste cuando estos mismos estudios eran revisados por expertos la PS solo se encontraba en un $29,3 \%$. Asimismo, solo el $8,3 \%$ de los estudios incluía los costes de productividad ${ }^{[8]}$.

En muchos países donde la EES es un método para la toma de decisiones se utiliza la PS como perspectiva de estudio, otros países utilizan solo la perspectiva del financiador y cada vez más países 


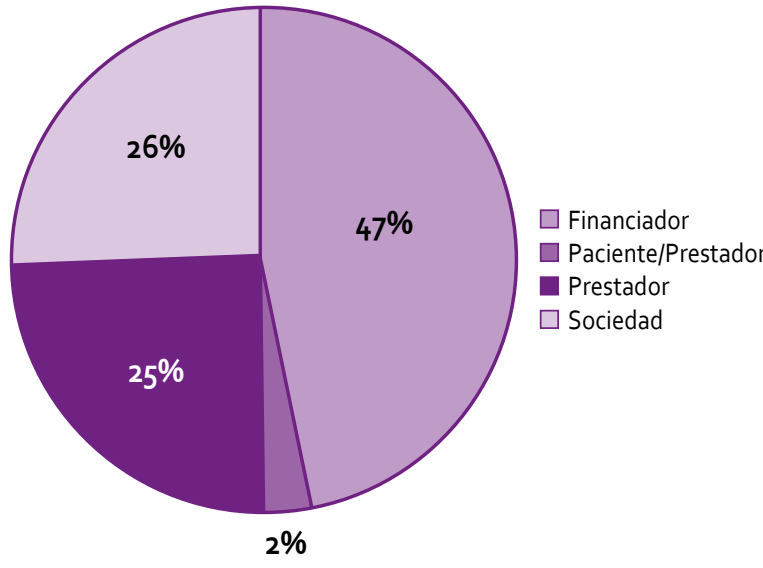

Figura 1. Perspectiva de las evaluaciones económicas en salud en el Perú.

optan por una doble perspectiva, de preferencia la perspectiva del financiador y la PS ${ }^{[9]}$.

En la actualidad se recomienda el desarrollo de la PS en las EES que complemente el análisis de las otras perspectivas y aporte información útil a los decisores ${ }^{[10]}$.

En ese sentido se realizó una búsqueda sistemática por pares de manera independiente en PubMed, Scielo y Google Scholar con las palabras clave: Economic Evaluation, cost effectiveness, cost benefit, cost utility, cost minimization, cost illness, cost analysis y Perú. La búsqueda incluyó todos los años en los que estuvieran disponibles estudios. Se excluyó estudios que aborden procedimientos administrativos y que no aborden específicamente intervenciones de salud. Se evaluó la metodología, buscando los costos incluidos en los estudios. En caso el estudio no explicitara la perspectiva se procedió al análisis de los costos incluidos; para definir que un estudio consideraba la PS debía incluir al menos los costos de productividad o los costos de cuidadores en el hogar. Se revisó los resultados de manera pareada, y las diferencias se solucionaron por consenso.

Se encontró 47 EES publicadas, entre estudios de costo efectividad $(42,6 \%)$, análisis o descripción de costos $(23,4 \%)$, estudios de costes de enfermedad (29,8\%), estudios de impacto económico $(2,1 \%)$ y estudios que incluían costes de enfermedad y coste efectividad (2,1\%). De los estudios hallados el $70,2 \%$ fueron publicados en los últimos 10 años, y el $38,3 \%$ fueron publicados en inglés.

La PS solo es usada en el $25,5 \%$ de los estudios evaluados y poco menos de la mitad de los estudios evaluados utiliza la perspectiva del financiador. (Figura 1)

La poca utilización de la PS subestima los costos asociados a la productividad, así como también no toma en cuenta los costos asociados al cuidado de las personas enfermas, esto, repercute directamente en el bienestar social de la gente. Estos costos de productividad son de importancia en las enfermedades crónicas degenerativas y discapacitantes ya que existe un costo de oportunidad para el ciudadano, este costo pudiera ser mayor que el costo sanitario directo en dichas enfermedades.

La dificultad en la estimación de los costos de productividad y la falta de data para la valoración de los costos sombra asociados al cuidado no remunerado de los enfermos son algunas de las barreras para la utilización de la PS.

En el país es necesario incorporar la PS en las EES para lo cual es imprescindible desarrollar los documentos técnicos y metodológicos para la elaboración de las EES que incluya los criterios para la estimación de los costos y las consecuencias desde la perspectiva social.

Fuente de financiamiento: Los autores declaran no haber recibido ninguna financiación para la realización de este trabajo.

Conflicto de intereses: Los autores declaran no tener conflicto de intereses con la publicación de este artículo.

\section{REFERENCIAS BIBLIOGRÁFICAS}

1. Husereau D, Drummond M, Petrou S, Carswell C, Moher D, Greenberg $\mathrm{D}$, et al. Consolidated Health Economic Evaluation Reporting Standards (CHEERS) statement - explanation and elaboration: a report of the ISPOR Health Economic Evaluation Publication Guidelines Good Reporting Practices Task Force. Value Health. 2013;16(2):231-50.

2. Loza C, Castillo-Portilla M, Rojas JL, Huayanay L. Principios básicos y alcances metodológicos de las evaluaciones económicas en salud. Rev Peru Med Exp Salud Publica. 2011;28(3):518-27.

3. Jonsson B. Ten arguments for a societal perspective in the economic evaluation of medical innovations. Eur J Health Econ. 2009;10(4):357-9.

4. Meltzer M. Introduction to health economics for physicians. Lancet. 2001;358(9286):993-8.

5. Johannesson $M$, Jönsson $B$, Jönsson $L$, Kobelt $G$, Zethraeus $N$ Why should economic evaluations of medical innovations have a Societal Perspective? London: Office of Health Economics; 2009.

6. Van den HoutWB. The value of productivity: human-capital versus friction-cost method. Ann Rheum Dis. 2010;69(Suppl I):i89-91.

7. Instituto de Evaluación Tecnológica en Salud. Manual para la elaboración de evaluaciones económicas en salud. Bogotá: IETS 2014 .

8. Neumann P. Costing and perspective in published cost-effectiveness analysis. Med Care. 2009;47(7 Suppl 1):S28-32.

9. Claxton K, Walker S, Palmer S, Sculpher M. Appropriate perspectives for health care decisions. CHE Research Paper 54 York, UK: Centre for Health Economics, University of York; 2010.

10. Sanders GD, Neumann PJ, Basu A, Brock DW, Feeny D, Krahn M, et al. Recommendations for conduct, methodological practices, and reporting of cost-effectiveness analyses: second panel on costeffectiveness in health and medicine. JAMA. 2016;316(10):1093103. 